\title{
Directed differentiation of human embryonic stem cells to neural progenitor cells and neurons on human feeder cells
}

\author{
Ding Yuan ${ }^{1,2, *}$, Pingyuan $\mathrm{Xie}^{1,2,{ }^{*}}$, Ge Lin ${ }^{1,2}$, Guangxiu Lu ${ }^{1,2}$ \\ ${ }^{1}$ Institute of Reproductive \& Stem Cell Engineering, Central South University; ${ }^{2}$ National Engineering \& Research Center of \\ Human Stem Cells, Changsha, Hunan, China
}

Human embryonic stem cells (hESCs) could provide available tools for neural development research and potential sources for cell replacement therapy. Traditionally, neural induction was intiated through the formation of embryoid bodies (EBs) and found that cells underwent a similar process as in vivo neural development from a primitive neuroepithelia stage_-"rosette" to a more definitive stage-neural tube-like structure. Recently some researchers reported the direct induction on mouse feeder cells. Our previous study showed that human feeder cells could well maintain the undifferentiated state of hESCs. In this study, we performed neural differentiation either directly on human feeder cells or through the formation of EBs to see if they had the same efficiency and underwent similar process of neural development. After 7 9 days, cells in both groups took on the appearance of "rosettes". Immunofluorescence indicated that rosettes were Pax6+, Musashi+, Nestin+ and Sox1-. Cell counting at day 10 indicated that Pax6+ cells in the direct induction group and EBs group was $83.1 \%$ and $57.5 \%$ respectively. The "rosettes" later turned into neural tube-like structures 5 7 days later, which were stained positive for Pax6, Musashi, Nestin and Sox1. Cell counting at day 16 indicated that cells in the direct induction group were 89.9\% Pax6+ and 74.5\% Sox1+, while cells in EBs group were $67.2 \%$ and $68.1 \%$ respectively. RTPCR showed that cells in both groups had similar gene expression profiles, with neural development related genes up-regulated and pluripotent genes down-regulated. We then concluded that hESCs could be induced into neural progenitor cells by both induction strategies, yet direct induction seemed to have a higher efficiency. In order to know the differentiating ability of "rosettes" obtained at day 10 and neural tube-like structures at day 16, we further induced these two distinct neural progenitors into midbrain dopaminergic neurons by adding FGF8 and SHH into the differentiating medium. Immunofluorescence at day 28 indicated both groups could differentiated into b-tubulin+ neurons, among which tyrosine hydrozylase positive cells in day 10 and day 16 group was $10 \%$ and $3 \%$ respectively. RT-PCR showed cells derived from day 10 had a higher expression of midbrain dopaminergic neuron marker En1 and Nkx6.1 than those from day 16. Thus the progenitors derived from "rosettes" could be more suitable for differentiation into midbrain dopaminergic neurons. We considered the direct differentiation performed on human feeder cells more approachable for further transplantation therapy.

Keywords: neural differentiation, progenitors, dopaminergic neurons

Cell Research (2008) 18:s146. doi: 10.1038/cr.2008.236; published online 4 August 2008

\footnotetext{
*These two authors contributed equally to this work

Correspondence: Guangxiu Lu

E-mail: dianayd66@yahoo.com.cn
} 